\title{
Applications of Coaxial Chokes to A-C Bridge Circuits
}

\author{
D. N. Homan* \\ Institute for Basic Standards, National Bureau of Standards, Boulder, Colo. 80302
}

(January 3, 1968)

\begin{abstract}
One or more turns of coaxial cable on a magnetic core form a coaxial choke which may be used to suppress unwanted loop currents in a-c bridge circuits. A review of several applications of coaxial chokes to a-c bridge circuits is presented. These applications include suppressing ground loop currents, assuring unique definition in the measurement of small 3-terminal capacitors, reducing stray magnetic field and reducing voltage drops in leads caused by large ground capacitance current. There is also a discussion of techniques for greatly increasing the effectiveness of the coaxial choke.
\end{abstract}

Key Words: Bridge, capacitance, coaxial cable, ground loop, inductance, magnetic core, stray magnetic field.

\section{Introduction}

Large improvements in stability have recently been made in commercial 3-terminal capacitance standards, and transformer-ratio-arm bridges of high precision have become available. The availability of such equipment may result in measurements of better precision in a greater number of laboratories. The accuracy of such measurements can frequently be improved by the use of coaxial chokes in appropriate parts of the bridge circuit $[1,2] .{ }^{1}$ Coaxial chokes have been used by workers in other fields as well-sometimes under different names, such as "cable chokes" [3] and "baluns" [4]. This paper gives a review of several applications of coaxial chokes as applied to a-c bridge measurements

Often a-c bridge circuits have components interconnected with coaxial cables to prevent undesired coupling between different parts of the circuit. Frequently, the physical layout permits more than one return path for the current to a given component. Such multiple returns have the effect of producing currents in the outer conductors of the coaxial cable which are not equal and opposite to the corresponding inner conductor currents. This departure from equality can be called a "net current." The elimination of the net current is a major problem in precision a-c bridge measurements, and one technique, that of using coaxial chokes, will be discussed here.

*Radio Standards Engineering Division, HF Impedance Standards, NBS, Boulder, Colo. 80302.

${ }^{1}$ Figures in brackets indicate the literature references at the end of this paper.
A coaxial choke is formed by winding one or more turns of coaxial cable around a high permeability toroidal magnetic core, as illustrated in figure 1. A higher impedance to net current can be obtained either by increasing the number of turns through the core or by increasing the size (or permeability) of the core. In the former case, the increased cable length results in increased cable impedance which may be undesirable. In the latter case, the increased cost may be undesirable. The self-inductance of either conductor of the coaxial cable is given approximately by

$$
L=\frac{4 \times 10^{-9} \pi N^{2} \mu A}{S}
$$

where $L$ is inductance in henrys, $N$ is the number of turns, $\mu$ the permeability, $A$ the cross-sectional area

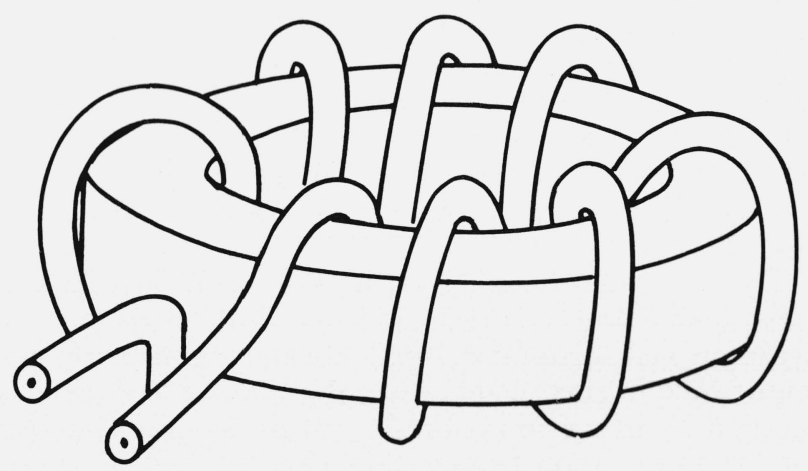

FIGURE 1. Coaxial choke (coaxial cable wound on a high permeability core). 


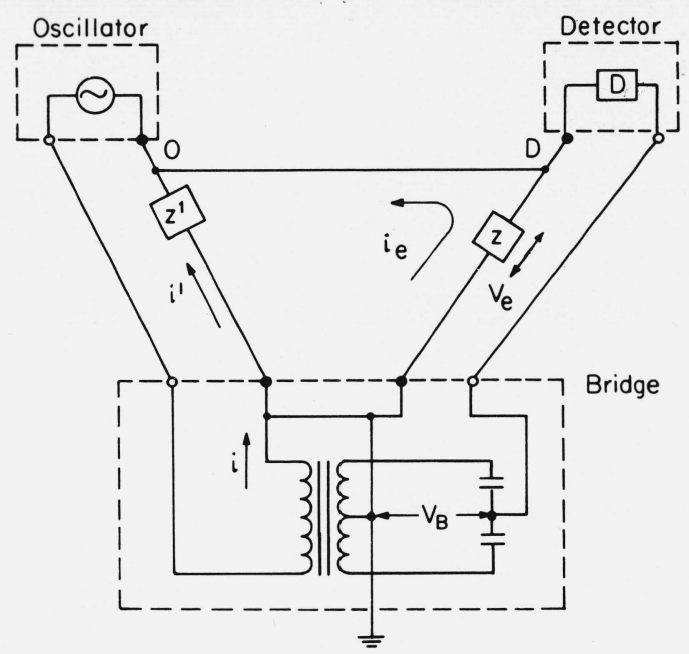

FIGURE 2. Capacitance measuring system showing ground loop.

of the core in $\mathrm{cm}^{2}$, and $S$ the mean core length in $\mathrm{cm}$. A typical range of inductance is 10 to $40 \mathrm{mH}$ at $1 \mathrm{kHz}$, for example. This inductance reduces only the net current in a coaxial cable; the equal "go and return" currents induce equal and opposite magnetic fluxes in the core. Thus, the contribution to the equivalent self-inductance from the high permeability core is zero for equal "go and return" current.

\section{Applications}

\subsection{Ground Loop}

If in the capacitance bridge illustrated in figure 2 [5] the connection OD were not present, the detector would sense $V_{B}$, the bridge unbalance voltage. However, if the case of the detector electrically contacts the case of the oscillator (connection OD), a ground loop is formed, accompanied by a possibility of an error in the measurement. The contact between oscillator and detector may be through a relay rack, or may result from both oscillator and detector being connected to a common power source (a-c line for example). Since $z^{\prime}$ is generally not zero, a portion of the current $i$ may be diverted through $z$, such that $i_{e}$ appears in the detector ground lead, resulting in a voltage drop $V_{e}$. In this case $V_{e}$ is an error voltage, adding to the bridge unbalance voltage $V_{B}$. When the detector indicates null, $V_{e}=V_{B}$, thus giving an erroneous result. In addition, the current $i_{e}$ in the loop completed by OD will generate a magnetic field which may couple into other parts of the circuit, or the loop may have voltage induced in it by other sources of magnetic field.

The error can be reduced by the use of low impedance leads and connectors from the bridge to the detector and generator, e.g., coaxial cable with low impedance in the shield. Also, the ground loop can be avoided by using an insulated, battery-operated detector, or an isolation transformer can be used in an appropriate place in the circuit. However, in cases where it is not convenient or possible to use an insulated,

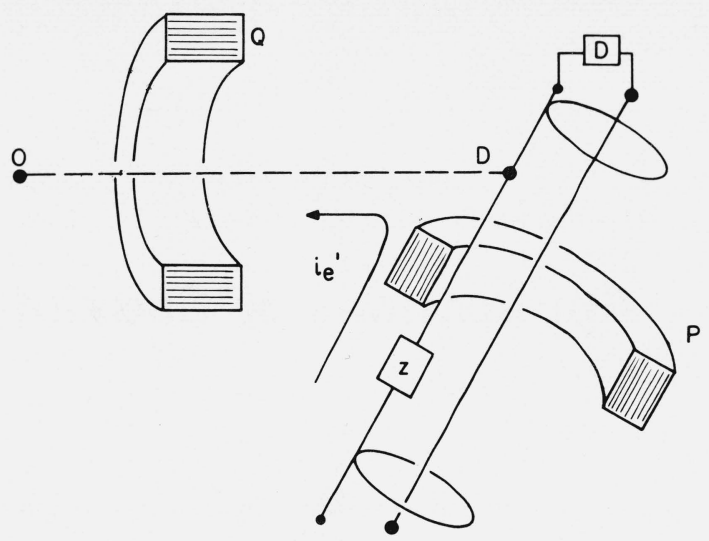

FIGURE 3. Partial circuit showing detector cable passed through a high permeability toroidal core.

battery-operated detector, or an isolation transformer, a coaxial choke in the coaxial cable between the bridge and the detector may achieve the desired result. A coaxial choke can also serve as a convenient test for trouble. A change in the bridge balance accompanying insertion of a coaxial choke indicates that a problem previously existed.

Figure 3 illustrates the use of the coaxial choke in the detector cable. It may be useful first to think of the toroid being around the connection OD in position Q. The single lead choke thus formed is a high impedance relative to $z$, so that $i_{e}^{\prime}$ in figure 3 is much less than $i_{e}$ in figure 2 (where no choke is used). Thus, the error voltage is considerably reduced; for example, if $z=z^{\prime}=0.01 \Omega$ and the impedance of the choke is $100 \Omega$, then $\frac{i_{e}^{\prime}}{i_{e}} \cong 2 \times 10^{-4}$. One can now imagine pushing the magnetic toroidal core into position $\mathrm{P}$ around the detector cable; the two positions are electrically equivalent.

It may be useful, as a rule of thumb, to consider the use of a coaxial choke in every unwanted loop in the measuring circuit. For $N$ paths to some reference point it may be necessary to use $N-1$ coaxial chokes.

\subsection{Measurement of a 3-Terminal Capacitor}

A 3-terminal capacitor connected to a bridge is illustrated in figure 4 . Here $C_{1}$ is the capacitance to

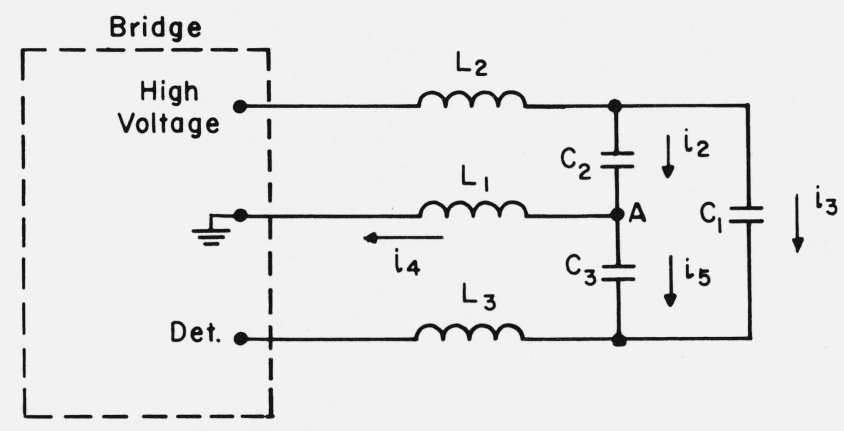

FIGURE 4. 3-Terminal capacitor connected to a bridge with leads having inductances $\mathrm{L}_{1}, \mathrm{~L}_{2}$ and $\mathrm{L}_{3}$. 


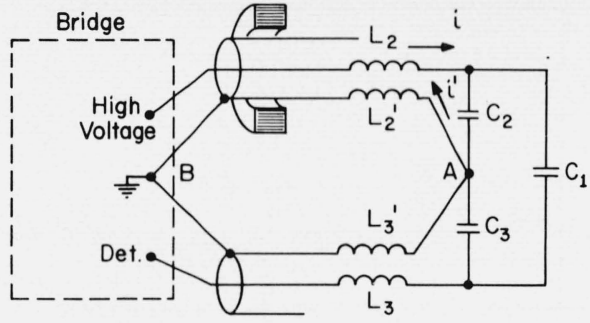

Figure 5a. 3-Terminal capacitor connected to a bridge showing the use of a coaxial choke.

be measured; $C_{2}$ and $C_{3}$ are capacitances to ground; $L_{1}, L_{2}$, and $L_{3}$ are lead inductances. It has been shown [6] that the effective capacitance seen by the bridge is given by

$C_{\text {eff }} \cong C_{1} \cdot\left[1-\frac{\omega^{2} C_{2} C_{3}}{C_{1}} L_{1}+\omega^{2} C_{2} L_{2}+\omega^{2} C_{3} L_{3}\right.$

$$
\left.+\omega^{2} C_{1}\left(L_{2}+L_{3}\right)\right]
$$

If $C_{1}$ is small, relative to $C_{2}$ and $C_{3}$, then

$$
C_{\mathrm{eff}} \cong C_{1}\left[1-\frac{\omega^{2} C_{2} C_{3} L_{1}}{C_{1}}\right] .
$$

For example, $\left|\frac{\omega^{2} C_{2} C_{3} L_{1}}{C_{1}}\right|$ can be $10^{-5}$ if $C_{1}=1 \mathrm{pF}$, $C_{2}=C_{3}=1000 \mathrm{pF}, \omega=10^{4}$, and $L_{1}=0.1 \mu \mathrm{H}$. At point $\mathrm{A}, i_{2}$ is divided between $j \omega L_{1}$ and $\frac{1}{j \omega C_{3}}$. Thus the voltage developed across $L_{1}$ by the current $i_{4}$ drives current $i_{5}$ into $C_{3}$, and to the detector, though it did not pass through $C_{1}$, which is the capacitance being measured. The capacitor's apparent value therefore will depend upon the circuit in which it is measured, and thus will not be defined uniquely.

Let us assume that the leads from the active elec-

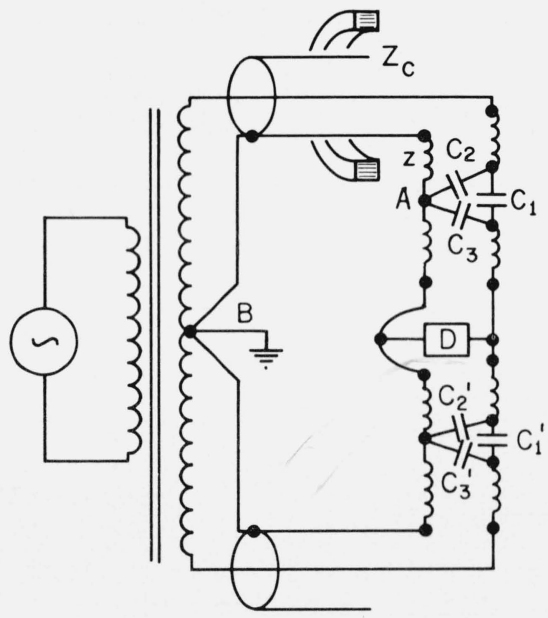

FIGURE 5b. Transformer bridge with coaxial choke.

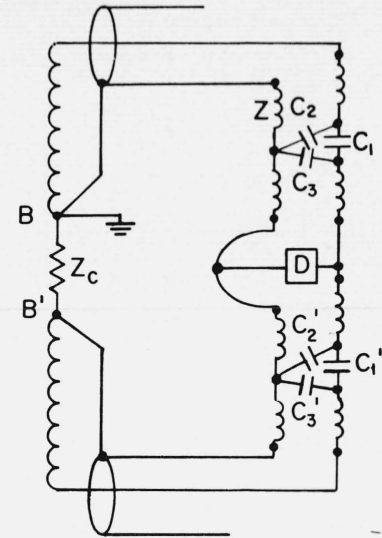

Figure 5c. Bridge split to show effect of the coaxial choke.

trodes of a 3-terminal capacitor are attached to the inner members of coaxial fittings, and that the case of the capacitor and the outer members of the coaxial connectors form the third "terminal." Also assume that such a capacitor is connected to a bridge with two coaxial cables. The effect that may cause an error (discussed in the preceding paragraph) can be nearly eliminated by inserting a coaxial choke into a cable connected to the capacitor as illustrated in figure $5 \mathrm{a}$. The choke is a high impedance to net current $\left(i-i^{\prime}\right)$. Thus most of the current in $C_{2}$ returns to ground through $L_{2}^{\prime}$, rather than through $C_{3}$ and $L_{3}$.

Figure $5 \mathrm{~b}$ shows a transformer-ratio-arm bridge as a practical example. Here $C_{2}, C_{3}, C_{2}^{\prime}$, and $C_{3}^{\prime}$ are ground capacitances. Lead inductances are also shown. The result of using the coaxial choke may be thought of as breaking the connection $\mathrm{BB}^{\prime}$ in figure $5 \mathrm{c}$ and inserting the high impedance, $Z_{c}$. The impedance of the ground loop is greatly increased by the core.

Figure 6 illustrates how the small inductance, $l_{1}$, of the case of the capacitor itself, in conjunction with ground capacitances $C_{2}$ and $C_{3}$, may cause the capacitance being measured to differ from the value calculated from the capacitor's dimensions. This difference would remain even if a coaxial choke of infinite impedance were used; it would be considered an error only in the case of a calculable capacitor and has, for example, been evaluated for the NBS cross capacitor [6]. Mention of it is included here only to show that not

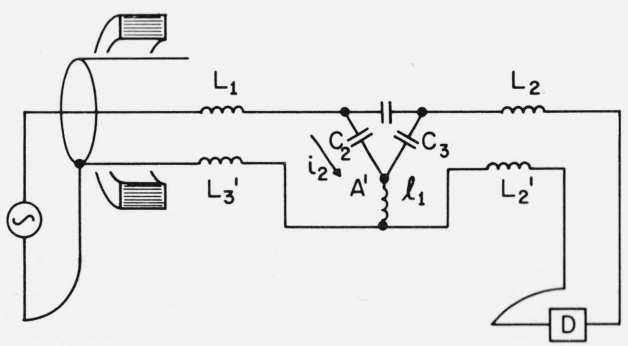

FIgURE 6. Partial bridge circuit to illustrate $\mathrm{l}_{1}$ a small inductance which is part of the capacitor, not the connecting leads. 


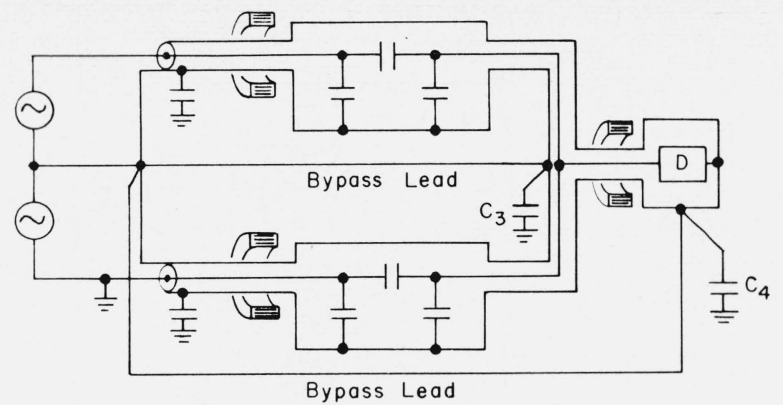

FiguRE 7. Circuit using bypass leads and coaxial chokes.

all errors of this type can be eliminated by simply using a coaxial choke. Current $i_{2}$ is divided at $\mathrm{A}^{\prime}$ between $j \omega l_{1}$ and $\frac{1}{j \omega C_{3}}$.

\subsection{Stray Magnetic Field}

A net current in any given coaxial cable of a bridge circuit flows in other conductors of the circuit. Because of the physical layout these other conductors have the effect of creating loops that are large relative to loops designed into the circuit. The magnetic field associated with the currents in such loops can cause trouble. A coaxial choke may be used to suppress the net current, thereby reducing the stray magnetic field.

However, if the net current to be eliminated is injected through a very high impedance, such as a small capacitor, the coaxial choke may be a relatively small impedance to the net current and nearly ineffective. The coaxial choke is an effective impedance to net current only when the net current is a result of a current divided by lead impedances that are small relative to that of the coaxial choke.

\subsection{Special Uses}

Figure 7 illustrates a technique used by Shields [7] to eliminate errors due to voltage drops in critical parts of his circuit. The voltage drops in the circuit were caused by the loading of capacitances $C_{3}$ and $C_{4}$. These are capacitances to ground from circuit shielding that was at a high potential above ground. The errors were eliminated by supplying current to the

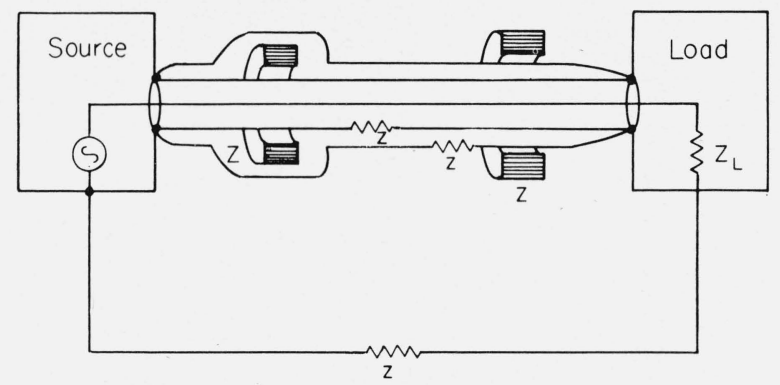

Figure 8. Use of triaxial cable and two coaxial chokes to multiply the effectiveness of the coaxial choke.

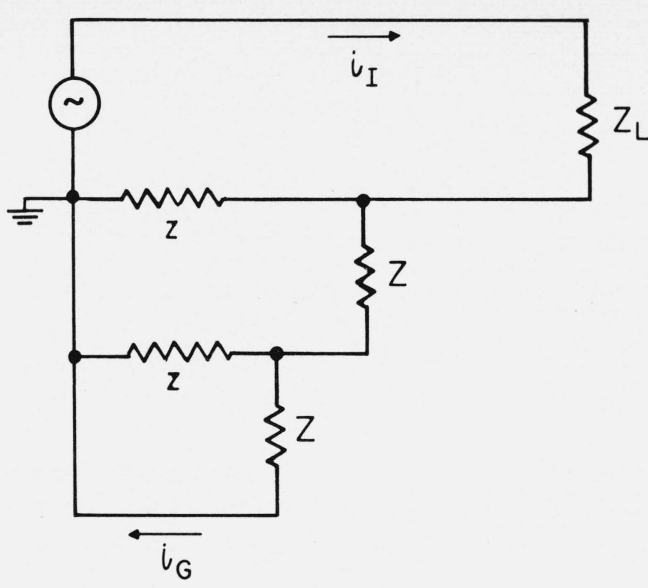

FIGURE 9a. "Triaxial choke" system with the outer shield completely enclosing the inner shield.

capacitances by low-impedance "bypass leads," and coaxial chokes were used in the coaxial cables as shown to restrict the current to the bypass leads in which voltage drops were not critical.

\subsection{Extra High Impedance Coaxial Chokes}

A coaxial choke can be modified to multiply its effectiveness by adding a shield and a magnetic core as shown in figure 8. Figure 9a is an equivalent circuit of figure 8. A calculation shows that such a "triaxial choke" can reduce net current by $\frac{z^{2}}{Z^{2}}$ where $z$ is the impedance of either shield and $Z$ is the impedance of each choke as shown in figure 8 . Since a single core coaxial choke reduces net current by approximately $\frac{z}{Z}$, a large improvement can be expected from the

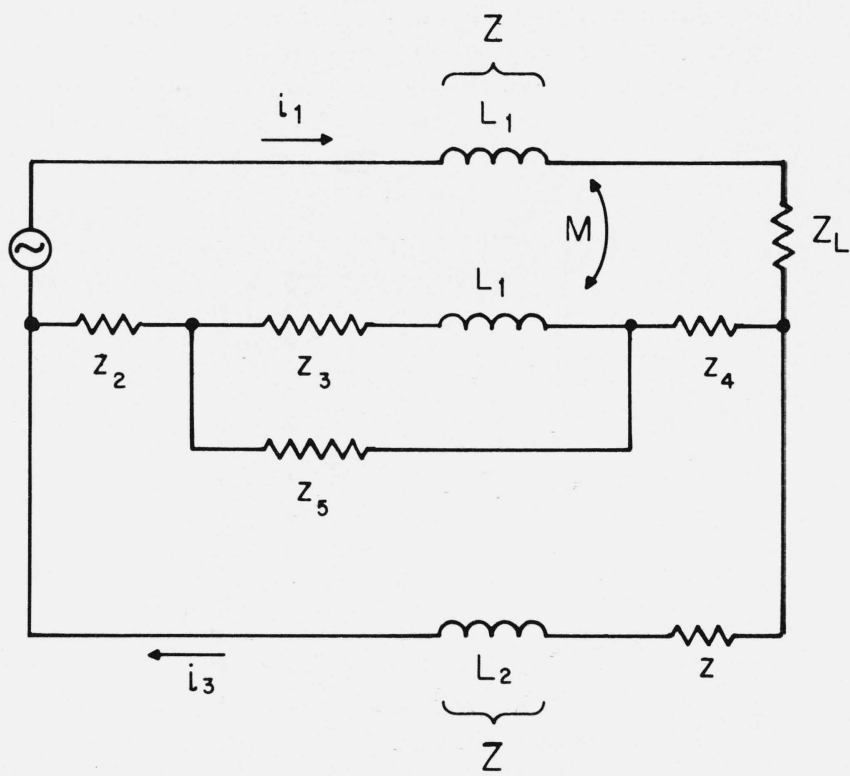

FIGURE 9b. Equivalent circuit of a "triaxial choke" system where outer shield is connected short of the ends of the inner shield. 


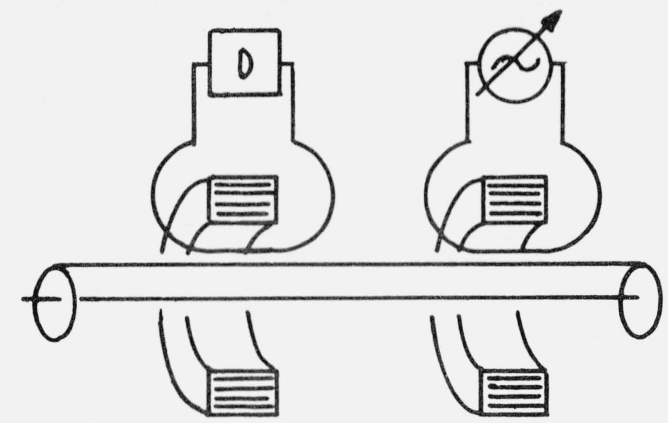

Figure 10. Manual high impedance coaxial choke.

"triaxial choke." To realize the maximum improvement from the "triaxial choke," care must be taken to connect the outer shield to the ends of the inner shield. If the outer shield is connected to the inner shield at points short of the ends, an equivalent circuit shown in figure $9 \mathrm{~b}$ can be used. A calculation based on this equivalent circuit shows that net current is reduced approximately by $\frac{z^{2}}{Z^{2}}+\frac{z_{2}+z_{4}}{Z}$, where the assumptions made are $z_{3}=z_{5} \equiv z, j \omega L_{1}=j \omega L_{2} \equiv Z$, and $z \ll Z$.

Two additional techniques $[8,9]$ for increasing the effectiveness of coaxial chokes also involve two cores, one a sensing core and the other a voltage injection core. One technique is to perform these functions manually as shown in figure 10. The detector senses flux in the core from net current in the coaxial cable. The generator output level can be adjusted such that the detector indicates null. The other technique is illustrated in figure 11 where the same functions are performed electronically. If $e_{p}=Z_{p} i, e_{Q}=G e_{p}$, and $\mathrm{e}_{T}=e_{p}+e_{Q}$, it can be shown that $Z_{T}=Z_{p}(1+G)$. The gain $G$ of the amplifier and the number of turns through the cores can be adjusted to reduce net current by an amount which will make the error current negligible.

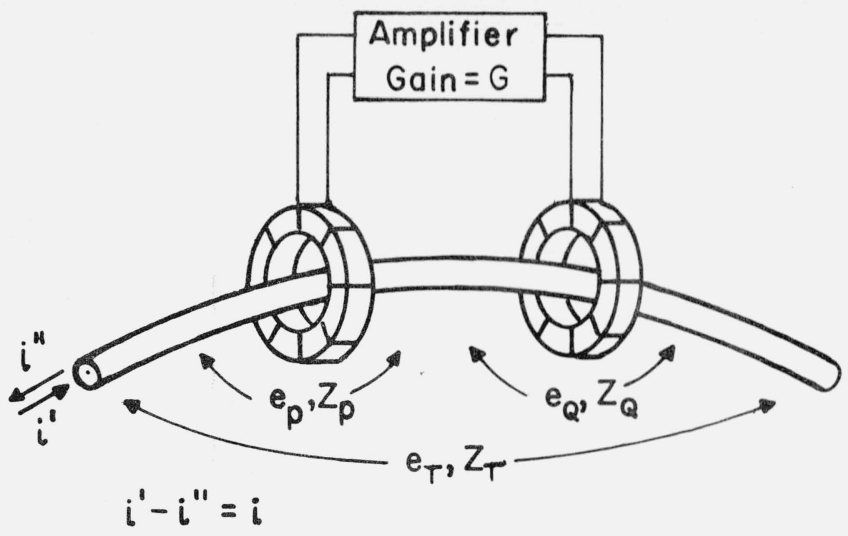

FIGURE 11. Active high impedance coaxial choke.

The author acknowledges the helpful suggestions of R. D. Cutkosky and J. Q. Shields.

\section{References}

[1] A. M. Thompson, The precise measurement of small capacitances, IRE Trans. on Instrumentation, 1-7, 3 and 4 (1958).

[2] A. M. Thompson, A-C bridge methods for the measurement of three-terminal admittances, IEEE Trans. on Instrumentation and Measurements, IM-13, 189 (Dec. 1964).

[3] E. T. Harper, Charting the bandwidth of isolating r-f chokes, Electronics, 39, 112 (June 13, 1966).

[4] T. A. Weil, Simple techniques to measure high-power, Electronic Design, 13, 42 (Nov. 8, 1965).

[5] Operating instructions for General Radio type 1615A capacitance bridge.

[6] R. D. Cutkosky, Evaluation of the NBS unit of resist based on a computable capacitor, J. Res. NBS 65A $\left(\mathrm{Ph}_{\mathrm{i}}\right.$ ics and Chemistry) No. 3, 147 (1961).

[7] J. Q. Shields, Voltage dependence of precision air capacitors, J. Res. NBS 69C (Engr. and Instr.) No. 4. 265 (1965).

[8] D. L. H. Gibbings, A circuit for reducing the exciting current of inductive devices, Proc. IEE, London, England, 108, Part B, 39 (1961).

[9] D. N. Homan, Some techniques for measuring small mutual inductances, J. Res. NBS 70C (Engr. and Instr.) No. 4, 221 (1966).

(Paper 72C2-274) 